\title{
EMILIO GARCÍA-SÁNCHEZ: LA MEJORA DE LA AUTONOMÍA DEL PACIENTE EN LA PRÁCTICA DE LA MEDICINA ESTÉTICA
}

\author{
THE IMPROVING OF THE AUTONOMY OF THE PATIENT IN THE \\ PRACTICE OF COSMETIC MEDICINE
}

Resumen: La reciente área de la medicina estética está suponiendo algunas novedades en la relación médico-paciente. Una de ellas consiste en que el "paciente estético" solicita al médico una intervención quirúrgica con una finalidad no siempre estrictamente terapéutica sino de tipo estética. El paciente quiere satisfacer el deseo de mejorar o transformar su belleza corporal y el médico ejecuta procedimientos para ofrecerle ese resultado. Para perfeccionar esa específica relación médico/paciente propongo pasar de un consentimiento informado como evento único plasmado en la firma de un papel, a un consentimiento que sea el resultado final de un proceso médico y ético en el tiempo que se establece entre el médico y el paciente. Dicho proceso se ajusta a lo que se denomina toma de decisiones informadas. Resulta eficaz para fortalecer el núcleo de la autonomía del paciente al perfeccionar su libertad racional para decidir someterse o no a la intervención estética, por otra parte no exenta de riesgos. Al mismo tiempo reporta beneficios para la salud y reduce el número de demandas a los médicos que suelen ser cada vez másfrecuentes en esta nueva área médica.

\begin{abstract}
The recent area of aesthetic medicine has brought some new features in the doctor-patient relationship. One is that the "aesthetic patient" asks the physiciana surgical procedure with a strictly non-therapeutic purpose but always aesthetic type. The patient wants to satisfy the desire to improve or transform your beauty body and the physician executesprocedures to offer that result.To improve that particular physician / patient relationship propose moving from informed consent as a single event reflected in the signing of a paper, a consent that is the end result of a medical and ethical process in time established between the doctor and the patient. This process conforms to what is called informed decisionsmaking.It is effective to strengthen the core of patient autonomy to perfect their rational freedom to decide whether or not to aesthetic intervention, on the other hand not without risks. At the same time it reported health benefits and reduce the number of lawsuits to doctors who tend to be increasingly frequent in this new medical field.
\end{abstract}

Palabras clave: medicina estética, autonomía del paciente, consentimiento

Keywords: aesthetic medicine, autonomy of the patient, informed consent

\section{Introducción}

La adecuada relación entre el médico y el paciente se encuentra en la 
base de la eficacia de la medicina. La reciente área de la medicina estética y cosmética ha supuesto algunas novedades en esa relación. A aparte de la avalancha de personas que está acudiendo a clínicas estéticas que demandan una mayor atención por parte de equipos especializados, hay una novedad en cuanto al tipo de solicitud del nuevo paciente. Ahora el nuevo "paciente estético" no solicita un tratamiento para remediar un problema objetivo de salud ya que se trata de un paciente que está sano. Solicita al médico una intervención quirúrgica de tipo invasiva 0 no invasiva de carácter exclusivamente estético. Por una preferencia personal el paciente desea mejorar su belleza corporal y el médico ejecuta procedimientos para ofrecerle ese resultado. Teniendo en cuenta la singular y a veces comprometida relación que se establece entre el médico y el paciente estético sugiero algunas medidas que pueden ayudar a mejorar la autonomía del paciente.

El aumento del número de consultas estéticas y a veces la falta de medios o de personal puede dificultar que el proceso informativo sobre las intervenciones sea completo y adecuado. La aceleración o la falta de rigor al informar pueden debilitar la confianza y la seguridad del paciente sobre el médico que va a llevar a cabo la intervención.Para perfeccionar esa específica relación propongo pasar de un consentimiento informado como evento único ${ }^{1}$ plasmado en la firma de un papel, a un consentimiento que sea el resultado final de un proceso médico y ético en el tiempo que se establece entre el médico estético y el paciente. Dicho proceso se ajusta a lo que se denomina toma de decisiones informadas, y protege eficazmente a los dos miembros que participan en él de las amenazas que se vierten hoy sobre las intervenciones estéticas.

Una de estas amenazas vadirigida a debilitar el núcleo de la autonomía y de la beneficencia, es decir, la libertad racional para elegir el tratamiento y el mejor interés del paciente. La otra amenaza se vierte sobre el propio prestigio del oficio médico tanto en su aspecto profesional como en su honestidad o calidad ética. La ciencia médica siempre ha gozado de autoridad y respeto social pero ahora tiene el riesgo de ver oscurecido el que ha sido siempre su esencial papel: curar, reestablecer la salud. La incorporación de la belleza como una nueva variable en la medicina está causando admiración y a la vez desconcierto en el mundo de la salud. La nueva medicina estética está proporcionando simultáneamente mucho bienestar y calidad de vida a un buen número de personas pero también daños a otras tantas. Sin duda se trata de una novedad porque esta ambivalencia en los resultados nunca se había dado tan marcadamente en otras áreas de la medicina. Para prestigiar más su trabajo sería deseable por parte de los médicos que conforman esta nueva área que arbitraran medidas que reduzcan el número de demandas que los pacientes ponen contra ellos y sus clínicas. En un buen número de casos, esas demandas han sido motivadas por una inadecuada información que les ha sido proporcionada acerca de los tratamientos o intervenciones a los que se

\footnotetext{
${ }^{1}$ De Roubaix JAM. Beneficence, non-maleficence, distributive justice and respect for patient autonomy e reconcilable ends in aesthetic surgery? Journal of Plastic, Reconstructive \& Aesthetic Surgery. 2011; 64, 11-16.
} 
sometieron.

Las dos sencillas sugerencias que propongo en este trabajo constituyen un camino pausado y virtuoso que deben recorrer conjuntamente médico y paciente. La meta es que el médico honesta y éticamente consiga una decisión informada, madura y libre del paciente que desee someterse a cirugías estéticas. El ejercicio de las virtudes del médico ${ }^{2}$ durante ese camino acaba enriqueciendo y fortaleciendo el principio bioético de la autonomía ${ }^{3}$ y su derechos a ella ${ }^{4}$.

\section{Conocer y comprender al paciente para involucrarlo en la toma de decisiones informada}

La toma de decisiones invoca a unacomunicación bidireccional entre los médicos y los pacientes, requerida para involucrar la preferencia del paciente en la elección eventual del tratamiento.Este proceso informativo recíproco pretende dilucidar si los beneficios y los posibles riesgos o efectos secundarios de una intervención estética corresponden con los deseos, valores y preferencias del paciente. ${ }^{5}$ El éxito del proceso consiste en construir conjuntamente la decisión entre los dos. El medico ha de facilitar que el paciente vaya elaborando sus reflexiones abiertamente en un clima de confianza. Como dice Kuczewski, sus recomendaciones pueden ayudar a que el paciente descubra sus propios valores y deseos e incluso a que con mayor consciencia termine de crearlos o de definirlos durante ese tiempo ${ }^{6}$.

Conocer al paciente de modo respetuoso implica saber qué es lo quiere realmente, qué piensa, cuáles son sus valores y sus preocupaciones reales, los motivos por los que desea operarse, sus expectativas etc. Resulta útil preguntarle si viene ya con alguna preferencia especial. Sin duda el médico ha de ejercitarse en escuchar para comprender. Comenta Epstein en su estudio que con frecuencia hay pacientes que se presentan en la consulta sin saber ni siquiera las preguntas adecuadas que deben hacer o sencillamente no se atreven a preguntar. Constituye un motivo más para que el médico se anticipe creando un clima de empatía y confianza ${ }^{7}$. Resulta aconsejable que el médico previamente a disponerse a ofrecer información sobre los procedimientos obtenga esa valiosa información. Se trata de un proceso deliberativo en el dónde el médico se convierte en un amigo más que en un consejero. A través del dialogo mutuo acaba evaluando el entendimiento del paciente y sus valores. La comunicación clara y fluida ayuda al autodesarrollo y la autodeterminación del paciente para que finalmente opte por la mejor de las

\footnotetext{
${ }^{2}$ Pellegrino EG, Thomasma, D. The Virtues in Medical Practice. New York: Oxford University Press, 1993.

${ }^{3}$ Beauchamp T, Childress J. Principles of biomedical ethics. Oxford University Press, New York, 2001.

${ }^{4}$ Ley $41 / 2002$, de 14 de noviembre, básica reguladora de la autonomía del paciente y de derechos y obligaciones en materia de información y documentación clínica.

${ }^{5}$ Ubbink DT, Santema TB, Lapid O. Shared Decision-Making in Cosmetic Medicine and Aesthetic Surgery. Aesthetic Surgery Journal. 2015; 1-6.

6 Kuczewski MG. Reconceiving the family: the process of consent in medical decisionmaking. The Hastings Center Report. 1996; vol 26, No.2, pp.30-37.

${ }^{7}$ Epstein RM, Alper BS, Quill TE. Communicating Evidence for Participatory Decision Making. JAMA. 2004; 291:2359-2366.
} 
opciones posibles ${ }^{8}$. Aquí el médico no impone nada, en todo caso le va persuadiendo delicadamente. Al final entre los dos consensuan la decisión como resultado de ese flujo recíproco informativo ${ }^{9}$.

Involucrar al paciente en este proceso puede requerir un plus de tiempo y de formación con respecto a otro tipo de consultas no quirúrgicas y no estéticas.Pero se ha comprobado que la adquisición de habilidades comunicativas y una adecuada formación del médico estético reducen objetivamente el tiempo de atención al paciente. Braddock III et al.,reconoce que estas habilidades tienen mucho que ver con aprender a comunicar y menos con la presión de tiempo a la que inevitablemente están sometidos los ocupados médicos y cirujanos estéticos ${ }^{10}$. Con frecuencia el tiempo se puede convertir en la excusa perfecta para no aplicar este procedimiento informativo sugerido. Es conocido y por otra parte comprensible que los cirujanos estéticos prefieran habitualmente estar más tiempo en el quirófano y menos en la consulta ${ }^{11}$.

Por propia experiencia todos sabemos que no somos capaces de recordar ni de asimilar mucha de la información que se nos da a los pacientes en la primera consulta médica que además suele ser corta. Precisamente en parte por este motivo compensa evitar las prisas para facilitar al paciente la mejor comprensión posible.

La eficacia y rapidez de este proceso requiere por un lado preparar previamente la conversación con el paciente a la vez que se selecciona la información para que cuanto antes ese paciente comprenda la intervención y se sienta involucrado en la toma de decisiones.Braddock $1 \mathrm{II}^{12}$ y Ubbink ${ }^{13}$ han comprobado que si el proceso informativo fue cuidadoso los pacientes al finalrequieren menos consultas de seguimiento y de aclaración, es decir menos tiempo total de discusión.Además, tras esta adecuada atención los pacientes reconocen que están mucho más seguros con la decisión final tomada. A esta seguridad se suma una mejoría en la satisfacción y en los resultados del paciente ${ }^{14}$. Guadagnoli y Ward incluso han comprobado que no solo se reduce la ansiedad, sino que además la recuperación es más rápida en comparación con aquellos pacientes que no se vieron implicados en la elección del procedimiento $^{15}$.

Es innegable que este proceso exige al médico un mayor esfuerzo por su parte y lo más cómodo sería no hacerlo. Pero si no hay un diagnóstico

\footnotetext{
${ }^{8}$ Emanuel EJ, Emanuel LL. Four models on the physician-patient relationship. JAMA. 1992. Vol.267, no.16, 2221-2226.

${ }^{9}$ Braddock III C, Hudak PL, Feldman JJ, Bereknyei S, Frankel RM, Levinson W. Surgery is certainly one good opinión: Quality and time-efficiency of informed decisión-making in surgery. J Bone Joint Surg Am. 2008; 90: 1830-8.

${ }^{10}$ Braddock III C, et al., op.cit., $1830-8$.

${ }^{11}$ Nejadsarvari N, Ebrahimi A. Different Aspects of Informed Consent in Aesthetic Surgeries. World J Plast Surg. 2014; 3(2):81-86.

12 Braddock III C, et al., op.cit., 1830-8.

${ }^{13}$ Ubbink DT, et al., op.cit., 1-6.

${ }_{15}^{14}$ Braddock III C, et al., op.cit., $1830-8$.

${ }^{15}$ Guadagnoli E, Ward P. Patient participation in decision-making. Soc. Sci. Med. 1998. vol. 47, no. 3, pp. 329-339.
} 
pausado y no se indaga en esos aspectos personales referidos se corren dos riesgos. Uno, que el médico condicione de modo paternalista al paciente y este acabe por elegir un tratamiento que no era el deseado o ajustado a sus valores $^{16}$; o que el paciente acabe tomando el control absoluto sobre la decisión optando como a veces sucede por alternativas que no son las más convenientes desde el punto de vista médico. En este último caso,paradójicamente, acabaría subyaciendo un concepto estrecho de la autonomía que conduce a que el médico prefiera no establecer una comunicación detenida con el paciente para no interferir en nada en sus valores y preferencias y de este modo no imponer su voluntad. ${ }^{17}$

Atiyeh y Emanuel aseguran que estas recomendaciones evitan el peligro real de convertir la relación médico estético- paciente estético en una relación técnico comercial en donde se informa de la técnica y de los precios. Creo que muchos buenos profesionales de esta área desean evitar la imagen externa que en ocasiones se da del médico estético como un mero proveedor y al paciente como un simple consumidor ${ }^{18}$.

\section{Ofrecer toda la alternativa posible incluida la no cirugía y el no tratamiento en absoluto}

En las definiciones que se suelen dar de consentimiento informado, al menos en la fase oral de esa información, se habla expresamente de que han de aparecer relacionadas todas las posibles alternativas al tratamiento elegido o sugerido.

Tal y como dice Rees en su estudio, la mayoría de pacientes que llegan a una clínica estética es más fácil que digan que sí a un procedimiento estético que decir no ${ }^{19}$. Por este motivo, el medico debería evitar sobreevaluar sus preferencias hacia unas alternativas en particular sobre las que se excede en pormenores en detrimento de otras alternativas que apenas comenta o que ni siquiera las ofrece. Esta actitud condiciona inevitablemente al paciente. El médico siempre alberga el temor de disuadir al paciente de la operación si le ofrece demasiada información alternativa ${ }^{20}$. Pero si él cede a la tentación de ocultar esa información está vulnerando la libertad y la autonomía porque priva al paciente del acceso a otras opciones legítimas y válidas.

Guadagnoli y Ward afirman que en general los pacientes desean y exigen ser informados de todas las alternativas de tratamientos. Quieren

\footnotetext{
${ }_{17}^{16}$ Emanuel EJ, Emanuel LL., op.cit., 2221-2226.

17 lbíd., 2221-2226; Ubbink DT, et al., 1-6.

${ }^{18} \mathrm{G}$. Maio, "Is aesthetic surgery still really medicine? An ethical critique", Handchir Mikrochir Plast Chir. 2007; 39: 189-94; Cf. Atiyeh BS, Rubeiz MT, Hayek SN. Aesthetic/cosmetic surgery and ethical challenges. Aesth Plas Surg.2008. 32: 829-839.

${ }^{19}$ Rees LS, Myers S, Bradbury E. A Comprehensive Screening, Education, and Training Tool for the Psychological Assessment of Patients Seeking Aesthetic Surgery: "DESIRABLE OP?" Aesth Plast Surg. 2012; 36:443-447.

${ }^{20}$ De Roubaix JAM. Beneficence, non-maleficence, distributive justice and respect for patient autonomy e reconcilable ends in aesthetic surgery? Journal of Plastic, Reconstructive \& Aesthetic Surgery (2011) 64, 11-16.
} 
participar en la toma de decisiones pero con la condición de disponer primero de la información de todas las opciones y las consecuencias de cada una ellas $^{21}$. Epstein va más allá insistiendo en que el derecho a la información de todos los posibles tratamientos es incluso independiente de si el paciente decide finalmente participar o no en la toma de decisiones, operarse o no. De tal modo, que esa información debe presentarse antes de fijar cual será el papel del paciente en la decisión ${ }^{22}$.

Resulta lógico que se revelen en primer lugar las alternativas de tratamientos más habituales con sus ventajas y desventajas, en su mayoría operaciones quirúrgicas. Pero después, el médico tiene la obligación depresentar y explicar al paciente las alternativas no quirúrgicas e incluso la alternativa de no someterse a ningún tratamiento en absoluto ${ }^{23}$, y cada una de ellas acompañada de su balance de riesgos y beneficios. El paciente tiene derecho a conocer también esas dos opciones especificadas, a valorarlas y a considerarlas como alternativas sobre la que también podría decidirse libremente. ${ }^{24}$

Comenta Little que la alternativa de un tratamiento no quirúrgico es lo suficientemente relevante para que no sea excluida de la información necesaria para el consentimiento ${ }^{25}$. Lo mismo podría decirse de la opción que no ofrezca ningún tratamiento, es decir: no hacer nada. Ofreciendo esas legítimas alternativas la medicina ayuda a contrarrestar la constricción a la que se ven sometidos los pacientes cuando algunos médicos les ofrecen un único modelo estético/cosmético que se alcanza a través de una única alternativa. Al igual que las opciones quirúrgicas médicamente indicadas, las no quirúrgicas y el no tratamiento tienen que entrar también en la categoría de opciones médicas. Incluso dice Little que estas últimas podrían considerarse más médicas - o más saludables-todavía que las quirúrgicas, ya que son alternativas prácticamente libres de riesgos ${ }^{26}$, y por tanto constituiría una irresponsabilidad médica no ofrecerlas.

Por ejemplo en determinados pacientes particularmente obsesionados con su imagen y con el riesgo de caer en una dismorfia o dismorfofobia, la alternativa médica no quirúrgica consistente en un tratamiento psicológico o psiquiátrico puede resultar de gran efectividad sobre el paciente. Asimismo, una cirugía bariátrica o liposucción en una persona con un poco de sobrepeso o una mamoplastia de aumento en el caso una mujer delgada, en ambos

\footnotetext{
${ }^{21}$ Guadagnoli E, Ward P., op.cit., 329-339.

${ }^{22}$ Epstein RM, et al., 291:2359-2366.

${ }^{23}$ O'Brien CM, Thorburn TG, Sibbel-Linz A, McGregor AD. Consent for plastic surgical procedures. J Plast Reconstruc Aesthet Surg. 2006. 59(9): 983-989; Ubbink DT, et al., 1-6; Newell BL. Informed consent for plastic surgery. Does I cut deeply enough? Journal of legal medicine. 2011. 32: 315-335; De Roubaix., op.cit., 11-16.

${ }_{24}$ Atiyeh BS, Rubeiz MT, Hayek SN. Aesthetic/cosmetic surgery and ethical challenges. Aesth Plas Surg.2008. 32: 829-839.

${ }^{25}$ Little MO. Cosmetic surgery, suspect norms, and the ethics of complicity. In: Parens E, Ed. Enhancing Human Traits: Ethical and Social Implications. Washington, DC: Georgetown University Press, (2000). p. 168.

${ }^{26}$ lbíd., p.168.
} 
casospodrían ser perfectamente sustituidas por una adecuada dieta alimenticia que ayudara a adelgazar en un caso y a aumentar de peso en el otro. Ubbink et al., ha comprobado que en el ámbito quirúrgico los pacientes tienden a preferir aquellas opciones de tratamientos menos invasivas ${ }^{27}$ pero solo después de que hayan sido apropiadamente informados de los diferentes tratamientos, de ahí la importancia de no descartar la información de ninguna alternativa ${ }^{28}$.

La posibilidad de rechazar todos los tratamientos y por tanto no hacer nada no debería resultar extraña o inaudita en los pacientes que acuden a la clínica estética. El desconocimiento de los riesgos de la operación deseada, o la consolidación de los valores y de la opinión a lo largo de un completo proceso informativo ${ }^{29}$ constituyen motivos suficientes para que finalmente el paciente decida con libertad no someterse a ningún tratamiento o prefiera retrasarlo temporalmente. Como concluye O'Brien, en este caso la justificación y exposición de la opción del no tratamiento en absoluto se convierte inesperadamente para algunos pacientes en la información más relevante de todo el proceso de cara a tomar la decisión final ${ }^{30}$. Sin duda puede convertirse en lo que más le ha ayudado a decidirse.

Las dos alternativas que estamos proponiendo que se incluyan en los consentimientos informados, en el caso de elegirse finalmente, representarían un éxito del proceso de la toma de decisiones informada y por tanto un victoria de la autonomía.Reflejan un ejercicio más perfecto de la autonomía del paciente.Por tanto, el médico si quiere respetar de un modo más pleno la autonomía ha de facilitar esas nuevas posibilidades médicas, aunque esto suponga ver disminuidos sus beneficios económicos. A cambio el gran beneficio incuantificable es el incremento de la libertad de elección del paciente.

\section{Conclusión}

Actualmente el proceso de la toma de decisiones compartidas e informadas se ha convertido en la mejor estrategia de atención al paciente en las consultas de cirugía estética cosmética, aunque desafortunadamente se está aplicando lentamente. Si tenemos en cuenta que lo que está en juego es la salud del paciente, resulta más difícil que el cirujano estético tenga conciencia de ese objetivo si no lleva a cabo este proceso de atención preoperatoria $^{31}$. Estoy convencido de que muchas de las decisiones tomadas inadecuadamente sobre intervenciones estéticas y con negativas consecuencias en la salud se podrían haber evitado si se hubiera seguido esta estrategia informativa. Sin duda perfeccionan el consentimiento informado y por la tanto la autonomía.

\footnotetext{
${ }^{27}$ Ubbink DT, et al., 1-6

28 Ruiz-Azarola A, Perestelo-Pérez L. Participación ciudadana en salud: formación y toma de decisiones compartida. Informe SESPAS 2012. Gac Sanit. 2012:26(S):158-161

${ }^{29}$ Ubbink DT, et al., op.cit., 1-6

${ }^{30}$ O'Brien CM, et al., op.cit., 983-989.

${ }^{31}$ Braddock III C, et al., op.cit., 90: 1830-8.
} 


\section{Bibliografía citada}

Atiyeh BS, Rubeiz MT, Hayek SN. Aesthetic/cosmetic surgery and ethical challenges. Aesth Plas Surg.2008. 32: 829-839

Beauchamp T, Childress J. Principles of biomedical ethics. Oxford University Press, New York, 2001.

Braddock III C, Hudak PL, Feldman JJ, Bereknyei S, Frankel RM, Levinson W. Surgery is certainly one good opinión: Quality and time-efficiency of informed decision-making in surgery. J Bone Joint Surg Am. 2008; 90: 1830-8.

De Roubaix JAM. Beneficence, non-maleficence, distributive justice and respect for patient autonomy e reconcilable ends in aesthetic surgery? Journal of Plastic, Reconstructive \& Aesthetic Surgery. 2011, 64, 11-16.

Emanuel EJ, Emanuel LL. Four models on the physician-patient relationship. JAMA. 1992. Vol.267, no.16, 2221-2226.

Epstein RM, Alper BS, Quill TE. Communicating Evidence for Participatory Decision Making. JAMA. 2004; 291:2359-2366.

Guadagnoli E, Ward P. Patient participation in decision-making. Soc. Sci. Med. 1998. vol. 47, no. 3, pp. 329-339.

Kuczewski MG. Reconceiving the family: the process of consent in medical decisionmaking. The Hastings Center Report. 1996; vol 26, No.2, pp.30-37.

Ley $41 / 2002$, de 14 de noviembre, básica reguladora de la autonomía del paciente y de derechos y obligaciones en materia de información y documentación clínica.

Little MO. Cosmetic surgery, suspect norms, and the ethics of complicity. In: Parens E, Ed. Enhancing Human Traits: Ethical and Social Implications. Washington, DC: Georgetown University Press, (2000). p.168.

Maio, G., "Is aesthetic surgery still really medicine? An ethical critique", Handchir Mikrochir Plast Chir. 2007; 39: 189-94.

Nejadsarvari N, Ebrahimi A. Different Aspects of Informed Consent in Aesthetic Surgeries. World J Plast Surg. 2014; 3(2):81-86.

O'Brien CM, Thorburn TG, Sibbel-Linz A, McGregor AD. Consent for plastic surgical procedures. J Plast Reconstruc Aesthet Surg. 2006. 59(9): 983-989.

Pellegrino EG, Thomasma, D. The Virtues in Medical Practice. New York: Oxford University Press, 1993.

Rees LS, Myers S, Bradbury E. A Comprehensive Screening, Education, and Training Tool for the Psychological Assessment of Patients Seeking Aesthetic Surgery: "DESIRABLE OP?" Aesth Plast Surg. 2012; 36:443-447.

Ruiz-Azarola A, Perestelo-Pérez L. Participación ciudadana en salud: formación y toma de decisiones compartida. Informe SESPAS 2012. Gac Sanit. 
FECHA DE ENVÍO DE ORIGINAL: 23.11.2016 FECHA DE ACEPTACIÓN: 25.11.2016.

DOI: $\underline{\text { http://dx.doi.org/10.12795/IETSCIENTIA.2016.i02.20 }}$

2012:26(S):158-161

Ubbink DT, Santema TB, Lapid O. Shared Decision-Making in Cosmetic Medicine and Aesthetic Surgery. Aesthetic Surgery Journal. 2015; 1-6. 